\title{
Mechanistic Insights of Seeded Diamond Growth from Molecular Precursors
}

Christopher P. Ender ${ }^{\ddagger a}$, Jiaxu Liang ${ }^{\ddagger}$, Jonas Friebel ${ }^{a}$, Todd Zapata $^{a}$, Manfred Wagner $^{a}$, Anna Ermakova ${ }^{a}$ and Tanja Weila,*

aMax Planck Institute for Polymer Research, Ackermannweg 10, 55128 Mainz, Germany.

${ }^{\ddagger}$ denotes equal contribution

Correspondence to be addressed to: weil@mpip-mainz.mpg.de

ABSTRACT. We report mechanistic insights of the bottom-up synthesis of nanodiamonds (NDs) from adamantane derivatives as molecular precursors. Thermal cracking of tetracosane below the decomposition temperature of adamantane, 1-adamantylamine or 2-azaadamantane revealed the initial steps of seeded diamond growth mechanism based on pyrolysis studies.

\section{INTRODUCTION}

Over the past decades, nanodiamonds (NDs) and especially fluorescent nanodiamonds (FNDs) have received growing interest in various disciplines such as cell biology ${ }^{1}$, biomedical research ${ }^{2}$, and sensing ${ }^{3}$. They provide a number of superior properties compared to other nanoparticles due to their inert and transparent $\mathrm{sp}^{3}$ carbon lattice and the presence of color centers. ${ }^{4}$ Atomic defects like the negatively charged nitrogen-vacancy (NV-) center, where a nitrogen replaces a carbon atom that is situated next to a vacancy in the diamond lattice, provides remarkable optical and spin properties. ${ }^{5,6}$ Thus, defect engineering in FNDs is of great importance for achieving the desired properties. 
Commercially available NDs are commonly produced via detonation techniques where explosives like TNT and RDX detonate in a closed reaction chamber. ${ }^{7,8}$ Detonation nanodiamonds (DNDs) require complex purification steps and show a strong tendency to form clusters and nonuniform morphology, which complicates further functionalization and applications. ${ }^{5,8}$ Currently, the most prominent large-scale high pressure high temperature (HPHT) technique for FND synthesis requires milling of micron-sized diamonds. ${ }^{9}$ However, this technique only gives limited control over defects in single FNDs, their type, number, and quality, especially for sizes below $10 \mathrm{~nm}$ due to the harsh synthesis conditions. ${ }^{10}$ It was proposed that diamondoid molecular precursors could substantially reduce the transformation barrier for diamond synthesis compared with conventional carbon allotropes owing to the full $\mathrm{sp}^{3}$ hybridization of diamondoids and their similar structure to bulk diamond. ${ }^{11}$ For example, a recently reported HPHT approach to directly synthesize NDs from molecular precursors uses halogenated adamantane species to further reduce the harsh reaction conditions during HPHT synthesis. ${ }^{12}$ Additionally, EKIMOV et al. demonstrated the fabrication of nitrogen-doped FNDs from N-containing precursors. ${ }^{13}$ By introducing more reactive linear hydrocarbons, synthesis of NDs from diamondoids could be achieved at even lower temperatures. ${ }^{14,15}$ This strategy shows great promise for bottom-up diamond synthesis designed at a molecular level.

However, the different roles of diamondoids and decomposable carbon sources are not fully understood. Diamondoid molecules have been demonstrated to play a key role in lowering the nucleation barriers during chemical vapor deposition (CVD) of diamonds, where diamondoids were considered as molecular seeds. ${ }^{16}$ In order to realize defect engineering in FNDs, a stable seed with pre-organized impurity atoms would be required. Pyrolysis experiments are usually 
employed to test the thermal stability of molecules and to study the mechanism of diamondoid formation. ${ }^{17}$ Due to their thermodynamically favored formation, diamondoids like adamantane are formed at high temperatures during the cracking of crude oil or as a result of thermal decomposition of $n$-alkanes. ${ }^{18,19}$ Moreover, because of the highly symmetric diamond-like structure, diamondoids exhibit high thermal stability. ${ }^{19}$ We would like to emphasize that the usage of the term "molecular seed" differs from the term "seed" in the classical nucleation theory. To date, the mechanism of so-called "seeded diamond growth" still remains unknown.

Herein, we demonstrate a mechanistic approach to elucidate the formation of NDs from diamondoid "seeds" in the presence of a decomposable carbon source. We present the cracking

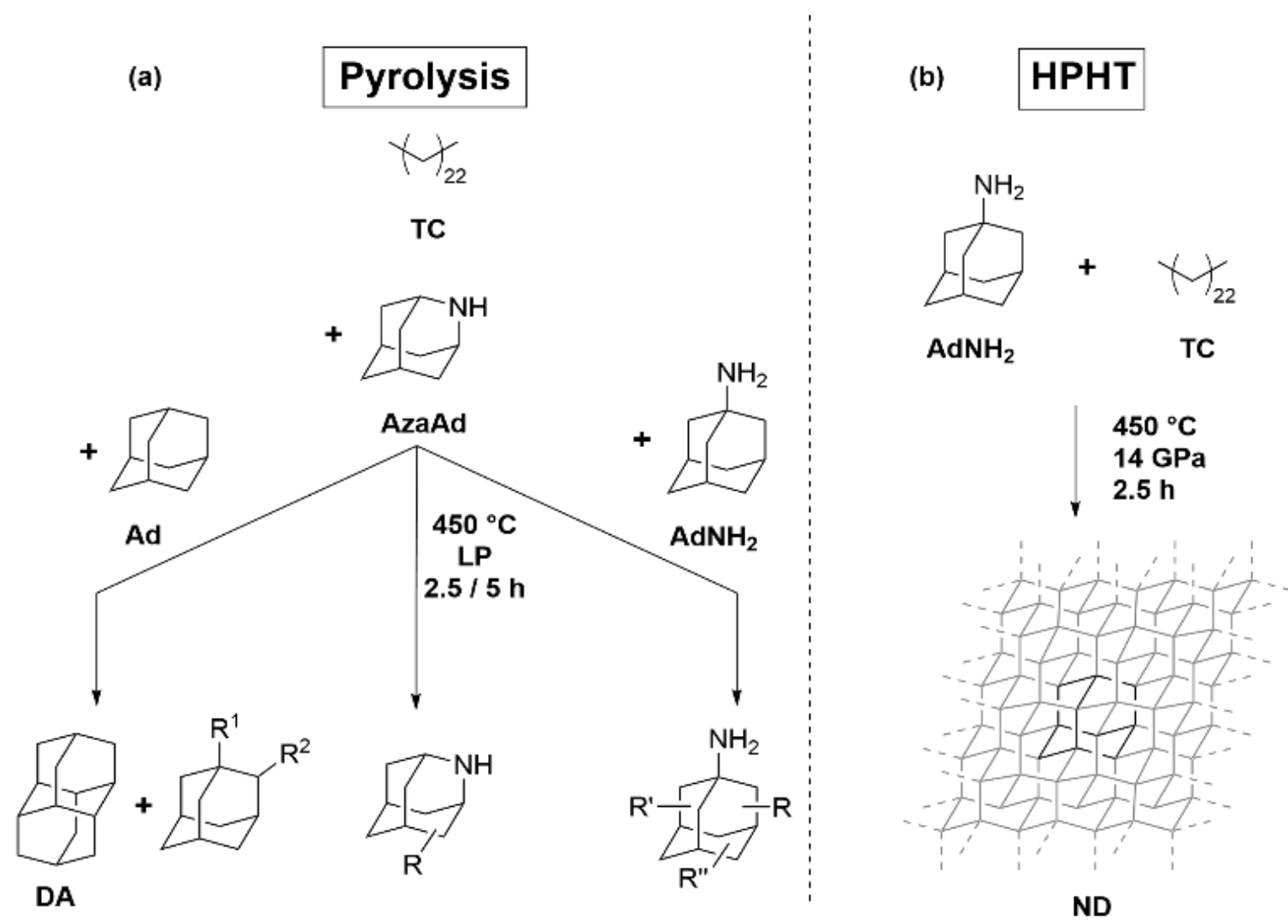

Figure 1: Overview of conducted experiments to investigate growth of diamonds from molecular precursors in the presence of a decomposable carbon source. (a) Pyrolysis experiments of mixtures of $\mathrm{Ad}$ and TC, AzaAd and TC and $\mathrm{AdNH} \mathrm{N}_{2}$ and TC at $450{ }^{\circ} \mathrm{C}$ and low pressure (LP). Pyrolysis was carried out for 2.5 or 5 h. (b) Nanodiamond (ND) synthesis from AdNH ${ }_{2}$ and TC under HPHT conditions $\left(450^{\circ} \mathrm{C}, 14 \mathrm{GPa}, 2.5 \mathrm{~h}\right)$. 
of tetracosane (TC) into lower molecular weight reactive hydrocarbons that react with the temperature-stable "seed" adamantane (Ad), 1-adamantylamine $\left(\mathrm{AdNH}_{2}\right)$, or 2azaadamantane (AzaAd), which we propose as the initiation step for ND growth under pyrolysis conditions (Figure 1a). Further, we demonstrate the synthesis of NDs based on the presented $\mathrm{AdNH}_{2} / \mathrm{TC}$ system (Figure $1 \mathrm{~b}$ ). We believe that our strategy will provide access to customized FNDs with precisely positioned lattice defects, pre-organized within the organic seed molecules, that remain intact during synthesis. Ultimately, such mechanistic studies will pave the way to FNDs with tailored optical or spin properties.

\section{RESULTS AND DISCUSSION}

Lately, seeded diamond growth using diamondoids as molecular precursors in presence of carbon sources has been reported in literature. ${ }^{14,15}$ It was proposed that the decomposable carbon source, e.g. TC, breaks under higher temperatures and builds up the diamond lattice on top of the diamondoid. In order to test this hypothesis, we conducted a series of pyrolysis experiments, which could reveal the initial states at the beginning of the diamond growth. First, pyrolysis of pure TC was conducted at temperatures between $380^{\circ} \mathrm{C}$ and $420^{\circ} \mathrm{C}$ to determine the fragments of the decomposition, which is important to understand the formation of reactive carbon species as the initial step of seeded ND growth. Equimolar amounts of TC were submitted into a glass ampoule, sealed under high vacuum and heat-treated for $2.5 \mathrm{~h}$. Afterwards, ampoules were opened and the extracted product was analyzed via gas chromatography-mass spectrometry (GC-MS). The pyrolytic products were identified to be lower molecular weight alkanes or olefins $\left(\mathrm{C}_{\mathrm{XH} 2 \mathrm{X}} / 2 \mathrm{X}+2, \mathrm{X}=10-21\right)$ as well as unreacted TC (Figure $\left.2 \mathrm{a} \& \mathrm{~S} 4\right)$. Their qualitative amount was 
determined by the integrated area (GC-MS) and was found to increase with higher temperatures. The ratio of the sum of pyrolysis products to the residual TC is only $12.3 \%$ at $380{ }^{\circ} \mathrm{C}$, whereas the ratio at $400{ }^{\circ} \mathrm{C}$ is $28.7 \%$ and increases again at $420{ }^{\circ} \mathrm{C}$ to $85.9 \%$ (Figure $2 \mathrm{~b}$ ). According to the decomposition of paraffins, e.g. $n$-heptane, the molecules undergo fission of $\mathrm{C}-\mathrm{C}$ bonds resulting in a radical-dominated mechanism. ${ }^{20}$ At higher temperatures, such as $420{ }^{\circ} \mathrm{C}, \beta$-elimination occurs as the predominant reaction leading to the dominant formation of $\alpha$-olefins over alkanes (Figure S5). Only at even higher temperatures and longer reaction times, the formation of aromatic species can be observed. ${ }^{20}$ Although treating TC at $450{ }^{\circ} \mathrm{C}$ for $5 \mathrm{~h}$ causes carbonization and aromatization, the main products are still linear fragments of TC (Figure S6).

Diamondoids like $\mathrm{Ad}, \mathrm{AdNH}_{2}$, or AzaAd (Figure 1) are commonly regarded as molecular seeds for seeded diamond growth. ${ }^{14,15}$ Therefore, we assessed the stability and reactivity of $\mathrm{Ad}, \mathrm{AdNH}_{2}$, or AzaAd at moderate temperature $\left(450{ }^{\circ} \mathrm{C}\right)$ in pyrolysis experiments. These experiments were carried out under low pressure and resulted in measured stabilities of $100 \%(\mathrm{Ad}), 93 \%\left(\mathrm{AdNH}_{2}\right)$, and 62\% (AzaAd), respectively (Figures S1, S2, S3). These results show that the cage structured diamondoids are relatively stable compared to the linear TC. When the diamondoid and TC were combined under the same reaction conditions, alkylation of $\mathrm{Ad} / \mathrm{AdNH}_{2} / \mathrm{AzaAd}$ occurred, clearly
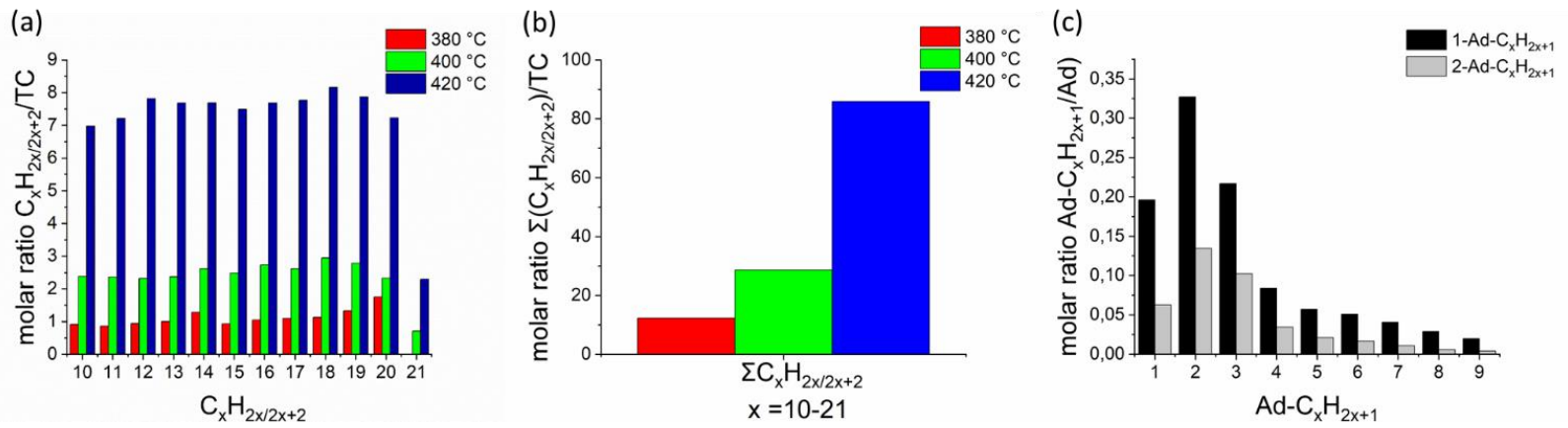

Figure 2: Qualitative analysis of GC-MS data obtained from pyrolytic experiments. (a+b) Pyrolysis of TC at different temperatures $\left(380-420^{\circ} \mathrm{C}\right)$. The molar ratio of alkyl fragments compared to the residual amount of TC (a). Sum of alkyl fragments versus temperature compared to TC (b). (c) Pyrolysis of a mixture of Ad and TC (molar ratio 1:1.1) at $450{ }^{\circ} \mathrm{C}$ (5 h). The plot shows the molar ratio of alkylated $\mathrm{Ad}$ at the bridgehead (1-Ad- $\left.\mathrm{C}_{x} \mathrm{H}_{2 x+1}\right)$ and the secondary position (2-Ad- $\left.\mathrm{C}_{x} \mathrm{H}_{2 x+1}\right)$. 
(a)

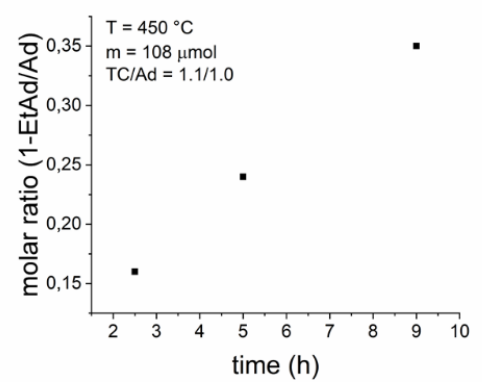

(b)

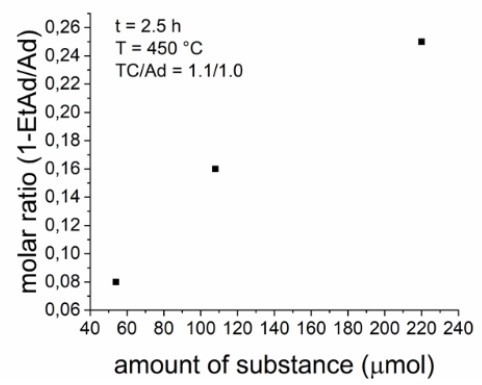

(c)

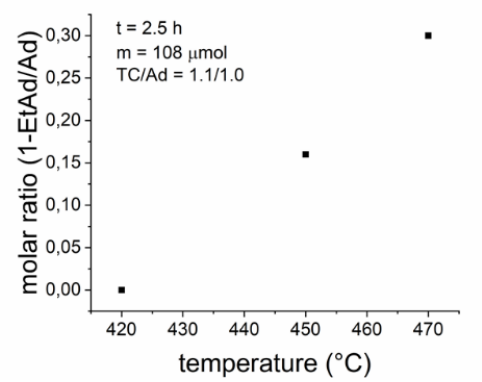

Figure 3: Pyrolysis study of a mixture of Ad and TC under different reaction parameters. (a) Variation of time. (b) Variation of the amount of substance. (c) Variation of temperature. Molar ratio of 1-Ethyladamantane to Ad serves as reference for all parameter studies.

supporting the formation of decomposing TC species and alkylation reactions occurring at the Ad scaffold. Alkylation of Ad can occur on two different positions, the secondary methylene position and the tertiary bridgehead position. Due to the slightly weaker $\mathrm{C}-\mathrm{H}$ bond, the reaction is favored at the bridgehead position. ${ }^{21}$ Nevertheless, the secondary $\mathrm{C}-\mathrm{H}$ bonds were also activated under pyrolysis conditions and substituted with alkyl species, which is crucial for the growth of nanodiamonds. Compared to the non-reacting and structurally unchanged Ad scaffold, GC-MS analysis of $\mathrm{Ad} / \mathrm{TC}$ mixtures shows high level of ethylated $\mathrm{Ad}$ species at 1- and 2-position (Figure $2 c+59)$. Therefore, the ratio 1-ethyladamantane to Ad serves as a reference to compare further experiments under variation of different parameters as it constitutes the highest proportion for all pyrolysis experiments. Figure 3 shows the results of the variation of parameters during pyrolysis of an Ad/TC mixture. With increasing time, higher temperature, and larger amount of substance, the ethylation of Ad increases, whereas the ratio of Ad to TC does not affect the result significantly (Figure S12). These results indicate that the reaction of Ad and TC performs best at temperatures between $450-470{ }^{\circ} \mathrm{C}$. Additionally, we propose the formation of the higher diamondoid diamantane during pyrolysis of the Ad and TC mixture, which could suggest the initiation of diamond growth. (Figure S10+11). We assume that TC breaks under the 
high temperature forming radicals reacting with Ad "seed" molecules thus initiating nanodiamond growth. In order to incorporate nitrogen defects into the diamond lattice, "seed" molecules with a heteroatom could be useful. ${ }^{15} \mathrm{AdNH}_{2}$ and $\mathrm{AzaAd}$ are such $\mathrm{N}$-containing compounds which could serve as precursors for later NV generation. Investigation of the growth mechanism of $\mathrm{AdNH}_{2}$ and TC was conducted at $450{ }^{\circ} \mathrm{C}(2.5 \mathrm{~h})$. Due to the amine function, the products obtain higher polarity compared to the aliphatic Ad derivatives. Thus, pyrolysis mixtures were separated via thin-layer chromatography (TLC, eluent $\mathrm{CHCl} / \mathrm{MeOH} / \mathrm{NH}_{3}$ 9:1.4:0.14) and subjected to atmospheric pressure chemical ionization-mass spectrometry (APCl-MS) (Figure S7 \& S8) as GC-MS is not applicable for characterization. The mass spectrum of $\mathrm{AdNH}_{2}$ with characteristic peaks of $[\mathrm{M}+\mathrm{H}]^{+}$and $[\mathrm{M}-\mathrm{NH} 3]^{+}$could be detected in spot (a) of the TLC plate. Additionally, APCI-MS of the second spot (b) shows the presence of several alkylated species of $\mathrm{AdNH}_{2}$. Next, pyrolysis of AzaAd and TC was conducted to elucidate the mechanism of diamond growth. Analysis via TLC and APCI-MS resulted in the formation of several alkylated species ranging from $C_{2}$ to $C_{11}$ alkane chains underlining the findings of the $A d / T C$ experiments. Here, it is not possible to differentiate between the alkylation patterns as a di-methylated $\mathrm{AdNH}_{2} / \mathrm{AzaAd}$ has the same mass as the single ethylated compound. Taking the results of Ad/TC into consideration, ethylation might be the favoured reaction compared to other alkylations. Our results support the observations of HEMMER et al. that HPHT treatment of AzaAd in combination with a carbon source affords FNDs. ${ }^{15}$

Next, ND synthesis under HPHT conditions was investigated using a diamond anvil cell. To proof the mechanistic findings of the pyrolysis study, an experiment was conducted on a mixture 

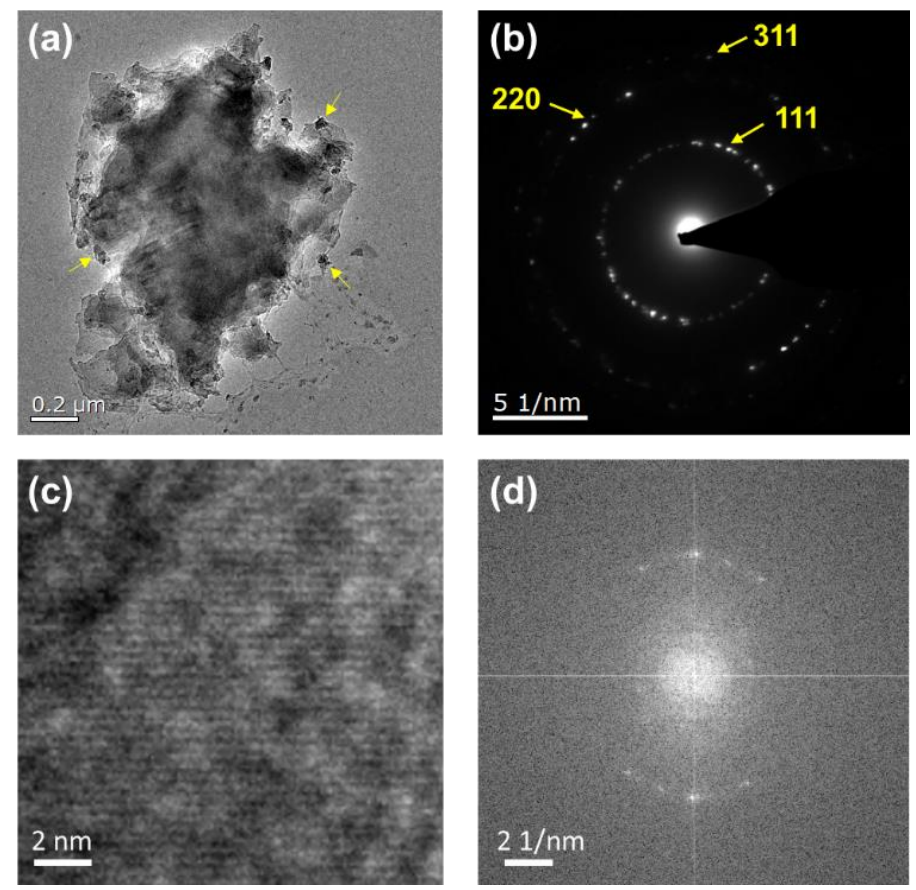

Figure 4: TEM measurements of the recovered product. (a) TEM image of diamond clusters; (b) electron diffraction pattern exhibiting lattice spacings of 2.07, 1.26, and $1.08 \AA$ A corresponding to diamond (111), (220) and (311) planes; (c) a high resolution image; (d) fast Fourier transform calculated from image (c).

of $\mathrm{TC}$ and $\mathrm{AdNH}_{2}$ at a molar ratio of 3:1 (Figure 1b). In the experiment, the initial pressure of $14 \mathrm{GPa}$ was applied and the sample was exposed to temperatures of $450{ }^{\circ} \mathrm{C}$. After the heating cycle, products of white color were obtained, indicating a large amount of organic residue. In line with previous reports ${ }^{22}$, the Raman spectra before and after the heating cycle under high pressure (Figure S13) only reveal minor differences indicating that the products are composed of similar linear aliphatic structures or unreacted TC as demonstrated in the pyrolysis experiments. Thereafter, product extraction proceeds on a grid treated at $200{ }^{\circ} \mathrm{C}$ in air to remove the organic residue before transmission electron microscopy (TEM) imaging. Small clusters of nanodiamonds were found along with large bulk of carbon due to the pyrolysis of organic materials (Figure 4). The selected area electron diffraction pattern showed reflections at $2.07,1.26$, and $1.08 \AA$ corresponding to Miller indexes of (111), (220), and (311), clearly confirming the diamond lattice. 
High resolution TEM images revealed crystalline lattice fringes with a d-spacing of approximately $2.07 \AA ̊$ confirmed by fast Fourier transform (FFT) images corresponding to the (111) plane of diamond lattice. These results indicate that nanodiamonds can be formed from the mixture of $\mathrm{AdNH}_{2}$ and TC at a relatively low temperature under high pressure. Compared with the results from the low pressure pyrolysis experiments, one could speculate that alkylation could be the first step towards diamond growth, which proceeds more completely at high pressures. The cage structure of $\mathrm{AdNH}_{2}$, which provides a similar diamondoid structure as bulk diamond, could reduce the energy barrier for diamond formation and serves as a molecular "seed" for diamond growth.

\section{CONCLUSIONS}

We have analyzed the processes for ND growth under pyrolysis conditions and transferred the findings to HPHT synthesis of NDs in the DAC. We demonstrated that TC breaks down and the formation of alkylated $\mathrm{Ad} / \mathrm{AdNH}_{2} / \mathrm{AzaAd}$ and potentially diamantane species were identified by GC-MS and APCI-MS. Based on our results, we propose that the seeded growth of NDs under HPHT requires decomposition of a carbon source like TC and the thermal stability of the cage structure of diamondoid seeds. We believe that ND growth is a consequence of TC cracking into reactive hydrocarbons of lower molecular weights, which react with the diamondoid lattice that pre-organizes them under the HPHT reaction conditions. By further optimizing the molar ratio of the precursors and HPHT conditions, we envision controlling the sizes of the NDs. Furthermore, synthesis of organic seeds with defined chemical structures could afford the controlled growth of versatile types of FNDs by incorporating heteroatoms like $\mathrm{N}, \mathrm{Si}$, and Ge. Production of FNDs with pre-organized lattice defects consequently provides a possibility to transform multiple fields which require nano-metrology techniques. 


\section{CONFLICTS OF INTEREST}

There are no conflicts to declare.

\section{ACKNOWLEDGEMENT}

Funding from the European Union's Horizon 2020 Research and Innovation Program under FETOPEN grant agreement no. 858149 "AlternativesToGd" is gratefully acknowledged.

\section{REFERENCES}

1 T. Zhang, A. Neumann, J. Lindlau, Y. Wu, G. Pramanik, B. Naydenov, F. Jelezko, F. Schüder, S. Huber, M. Huber, F. Stehr, A. Högele, T. Weil and T. Liedl, J. Am. Chem. Soc., 2015, 137, 9776-9779.

2 Y. Wu, A. Ermakova, W. Liu, G. Pramanik, T. M. Vu, A. Kurz, L. McGuinness, B. Naydenov, S. Hafner, R. Reuter, J. Wrachtrup, J. Isoya, C. Förtsch, H. Barth, T. Simmet, F. Jelezko and T. Weil, Adv. Funct. Mater., 2015, 25, 6576-6585.

3 F. Dolde, H. Fedder, M. W. Doherty, T. Nöbauer, F. Rempp, G. Balasubramanian, T. Wolf, F. Reinhard, L. C. L. Hollenberg, F. Jelezko and J. Wrachtrup, Nat. Phys., 2011, 7, 459-463.

4 T. D. Merson, S. Castelletto, I. Aharonovich, A. Turbic, T. J. Kilpatrick and A. M. Turnley, Opt. Lett., 2013, 38, 4170.

5 Y. Wu, F. Jelezko, M. B. Plenio and T. Weil, Angew. Chemie - Int. Ed., 2016, 55, 6586-6598.

6 W. Liu, B. Naydenov, S. Chakrabortty, B. Wuensch, K. Hübner, S. Ritz, H. Cölfen, H. Barth, K. Koynov, H. Qi, R. Leiter, R. Reuter, J. Wrachtrup, F. Boldt, J. Scheuer, U. Kaiser, M. Sison, T. Lasser, P. Tinnefeld, F. Jelezko, P. Walther, Y. Wu and T. Weil, Nano Lett., 2016, 16, $6236-6244$. 
7 V. Y. Dolmatov, M. V. Veretennikova, V. A. Marchukov and V. G. Sushchev, Phys. Solid State, $2004,46,611-615$.

8 X. Duan, W. Tian, H. Zhang, H. Sun, Z. Ao, Z. Shao and S. Wang, ACS Catal., 2019, 9, 74947519.

9 J.-P. Boudou, P. A. Curmi, F. Jelezko, J. Wrachtrup, P. Aubert, M. Sennour, G. Balasubramanian, R. Reuter, A. Thorel and E. Gaffet, Nanotechnology, 2009, 20, 235602.

10 J.-P. Boudou, J. Tisler, R. Reuter, A. Thorel, P. A. Curmi, F. Jelezko and J. Wrachtrup, Diam. Relat. Mater., 2013, 37, 80-86.

11 S. Park, I. I. Abate, J. Liu, C. Wang, J. E. P. Dahl, R. M. K. Carlson, L. Yang, V. B. Prakapenka, E. Greenberg, T. P. Devereaux, C. Jia, R. C. Ewing, W. L. Mao and Y. Lin, Sci. Adv., 2020, 6, eaay9405.

12 E. A. Ekimov, M. V Kondrin, S. G. Lyapin, Y. V. Grigoriev, A. A. Razgulov, V. Kryvobok, S. Gierlotka and S. Stelmakh, Diam. Relat. Mater., 2020, 103, 107718.

13 E. A. Ekimov, O. S. Kudryavtsev, N. E. Mordvinova, O. I. Lebedev and I. I. Vlasov, ChemNanoMat, 2018, 4, 269-273.

14 J. Liang, C. P. Ender, T. Zapata, A. Ermakova, M. Wagner and T. Weil, Diam. Relat. Mater., $2020,108,108000$.

15 M. Alkahtani, J. Lang, B. Naydenov, F. Jelezko and P. Hemmer, ACS Photonics, 2019, 6, $1266-1271$.

16 M. A. Gebbie, H. Ishiwata, P. J. McQuade, V. Petrak, A. Taylor, C. Freiwald, J. E. Dahl, R. M. K. Carlson, A. A. Fokin, P. R. Schreiner, Z.-X. Shen, M. Nesladek and N. A. Melosh, Proc. Natl. Acad. Sci., 2018, 115, 8284-8289. 
17 J. E. P. Dahl, J. M. Moldowan, Z. Wei, P. A. Lipton, P. Denisevich, R. Gat, S. Liu, P. R. Schreiner and R. M. K. Carlson, Angew. Chemie - Int. Ed., 2010, 49, 9881-9885.

18 J. E. Dahl, J. M. Moldowan, K. E. Peters, G. E. Claypool, M. A. Rooney, G. E. Michael, M. R. Mello and M. L. Kohnen, Nature, 1999, 399, 54-57.

19 G. N. Gordadze and M. V. Giruts, Pet. Chem., 2008, 48, 414-419.

20 J. Ding, L. Zhang, Y. Zhang and K.-L. Han, J. Phys. Chem. A, 2013, 117, 3266-3278.

21 G. Yan, N. R. Brinkmann and H. F. Schaefer, J. Phys. Chem. A, 2003, 107, 9479-9485.

22 R. H. Wentorf, J. Phys. Chem., 1965, 69, 3063-3069. 